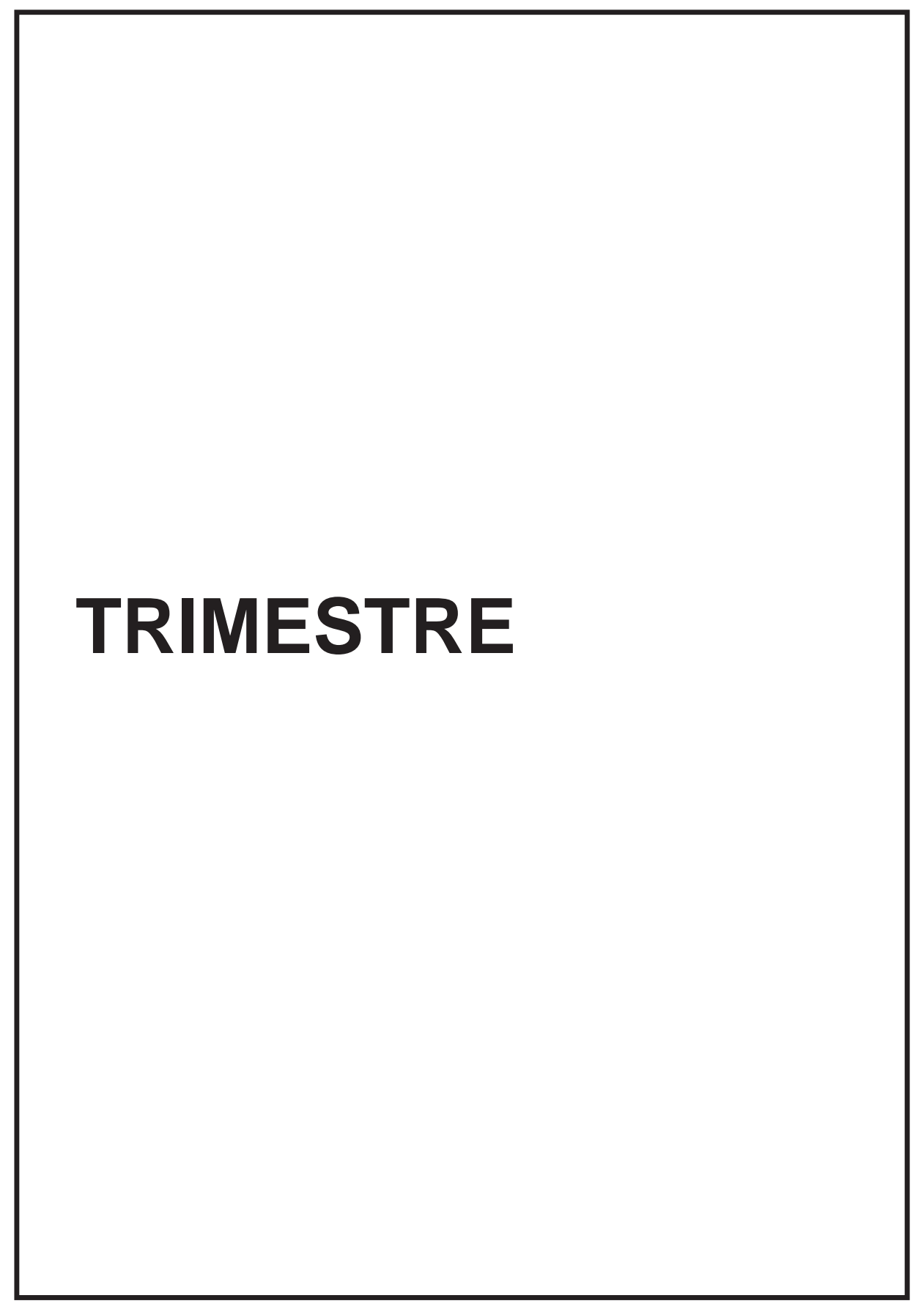




\title{
Factores clave de éxito en la gestión presupuestaria del sector pastas alimenticias en la región zuliana*
}

\author{
Rodríguez Medina, Guillermo** \\ Vílchez, Gil*** \\ Urdaneta Espinosa, Armando****
}

\section{Resumen}

La presente investigación tiene como propósito determinar los factores clave de éxito (FCE) en la gestión presupuestaria del sector pastas alimenticias en la región zuliana. Identificados éstos como los atributos que tienen las empresas con éxito, y de los cuales carecen las que no lo tienen. El trabajo se sustentó en autores como Welsch et al. (1994), Burbano y Ortiz (2000), Ormaechea et al. (1998), Horngren et al. (2002), entre otros. La investigación fue descriptivo de diseño no experimental y transeccional, basado en técnicas de recolección de datos primarias y secundarias tales como: Cuestionarios y entrevistas libres a operarios y empleados del sector, así como una revisión de diversos textos, y publicaciones internas de las empresas objeto de estudio. Los resultados indican que los FCE en la gestión presupuestaria del sector analizado se concentran en el área de costos de fabricación, la calidad en los procesos, la productividad y el tiempo de manufactura y entrega, permitiendo concluir que existen rasgos similares en la industria que apuntalan hacia un liderazgo en costos sustentado en un rendimiento eficiente de los procesos, permanente innovaciones tecnológicas e incremento del potencial productivo de las plantas procesadoras de pastas cortas y largas de la región.

Palabras clave: Factores clave de éxito, pastas alimenticias, gestión presupuestaria, costo, calidad.

Recibido: 06-04-18. Aceptado: 06-05-31

* Este artículo fue resultado del Programa de Investigación "Costos y/o Presupuestos Organizacionales" financiado por el Consejo de Desarrollo Científico y Humanístico de LUZ.

** Doctor en Ciencias. Mención Gerencia, Profesor Titular de la Universidad del Zulia (LUZ), Investigador del Centro de Estudios de la Empresa. E-mail grodriguezm@luz.edu.ve Autor para la correspondencia.

**** Especialista en Costos, Cursante de Doctorado en Ciencias Sociales. Profesor Titular de la Universidad del Zulia, Investigador del Centro de Estudios de la Empresa. E-mail: gilvilchez2001@yahoo.com.

***** Magíster en Gerencia de Empresas. Mención Financiera, Profesor Agregado de la Universidad Dr. Rafael Belloso Chacín, Asistente de Investigación del Centro de Estudios de la Empresa. E-mail: urdanetarmando@yahoo.com 
Factores clave de éxito en la gestión presupuestaria del sector pastas alimenticias

Rodríguez Medina, Guillermo; Vílchez, Gil y Urdaneta Espinosa, Armando.

\title{
Key Success Factors in Budget Management in the Noodle (Spaghetti) Industry in the Zulia Region
}

\begin{abstract}
The purpose of this research is to determine the key success factors (KSF) in the budgetary management of the spaghetti (pasta) industry in the Zulia region. Once these factors in successful noodle industry companies are identified, the lack of the same can be identified in unsuccessful companies. The study was based on authors such as Welsch et al. (1994), Burbano and Ortiz (2000), Ormaechea et al. (1998), and Horngren et al (2002), among others. The research was descriptive, nonexperimental and trans-sectional, and based on primary and secondary data collection techniques such as questionnaires, un-structured interviews with employees and operators in this industry, as well as a review of diverse texts and internal publications of the companies involved in the study. The results indicate that the KSF in budget management in the sector under study concentrates on production costs, process quality, productivity in relation to time and dispatch, all of which permits us to conclude that there are similar patterns in the industries under study that point to leadership in costs based on efficient processing, permanent technological innovation, and increased productive potential in spaghetti processing plants in the region.
\end{abstract}

Key words: Key success factors (KSF), spaghetti industry, budgetary management, costs, quality.

\section{Introducción}

Hoy en día las organizaciones precisan ineludiblemente cambiar para permanecer y lograr el éxito empresarial, en virtud que la alta rapidez y el marcado dinamismo denotado por el entorno económico venezolano a lo largo de los últimos años, ha propendido la necesidad que las empresas del sector pastas alimenticias deban ajustarse a nuevas condiciones del mercado.

Según Amat (1994:12), "La supervivencia y el éxito empresarial exigen una adaptación continua de la empresa al entorno tratando de lograr la máxima eficacia en el funcionamiento de su organización interna y de sus operaciones." Tal situación exige la adopción de un estilo estratégico de gestión, así como un control permanente de la evolución de la empresa para detectar posibles desviaciones respecto al funcionamiento adecuado y necesario.

De acuerdo a la situación planteada en el presente artículo es perentorio determinar los factores clave de éxito (FCE) en la gestión presupuestaria del sector de pastas alimenticias en la región zuliana, que está constituido por tres empresas. Sin embargo, además de su determinación y puesta en práctica, es necesario hacerles seguimiento a través del control y la evaluación continua para garantizar la supervisión operativa y financiera de los recursos asignados, y consecuentemente el sostenimiento de la rentabilidad económica de la empresa.

Sobre este particular Ormaechea et al. (1998) refieren que la gestión presu- 
puestaria, es un proceso sistemático a través del cual los gerentes encargados de la función de planeación ejercen influencia en otros miembros de la organización para lograr el cumplimiento de las estrategias previstas por la misma.

En la actualidad existe abundante información relacionada con la gestión presupuestaria, que desde una perspectiva metodológica autores como Welsch et al. (1994), Burbano y Ortiz (2000), Horngren et al. (2002), entre otros, permiten sustentar teóricamente el presente trabajo, así como precisar una diversidad de rasgos comunes presentes en experiencias exitosas en la gestión presupuestaria de las empresas traducidas en lo que se denomina en el ámbito gerencial "factores clave de éxito" (FCE). Ante tal particularidad se puede preguntar ¿Qué elementos se considerarían relevantes a la hora de identificar estos factores y cuáles serian estos factores? En efecto, lo que podría constituirse en un FCE para una industria en particular no necesariamente seria aplicable en otra; no obstante, existe la posibilidad de evidenciar características similares dentro de un mismo sector industrial que ofrezcan un perfil de los FCE determinantes en la gestión presupuestaria.

De acuerdo al problema planteado se consideró que el tipo de investigación es descriptivo, ya que se pretendió como manifiesta Méndez (1999:125) "la descripción de las características que identifican los diferentes elementos y componentes, y su interrelación, cuyo propósito es la delimitación de los hechos que conforman el problema de investigación".

Dado que enfrentaron una situación de cambio permanente y de optimi- zación de los procesos productivos, así como de una reestructuración de los servicios de soporte, tal situación permite el direccionamiento para el mejoramiento de la organización, por lo que surgen inquietudes que propician la necesidad de determinar qué FCE son considerados en la gestión presupuestaria por las empresas que operan en la industria manufacturera de pastas alimenticias en la región zuliana.

\section{Gestión Presupuestaria}

La unidad básica que sustenta estructuralmente una gestión presupuestaria evidentemente es el presupuesto, en tal sentido Mallo y Merlo (1995: 119) lo definen como, "(...) un medio de acción empresarial que permite dar forma en términos económicos a las decisiones contenidas en los planes y programas"; por su parte Welsch et al. (1994: 1) manifiestan que "es un enfoque sistemático y formalizado para el desempeño de fases importantes de las funciones administrativas de planificación y control"; mientras que Galindo y García (1987: 96) consideran que "es un elemento indispensable al planear, ya que a través de ellos se proyectan, en forma cuantificada, los elementos que necesita la empresa para cumplir con sus objetivos; sus principales finalidades consisten en determinar la mejor forma de utilización y asignación de los recursos, a la vez que controla las actividades de la organización en términos financieros".

De lo anterior, se desprende que la utilidad del presupuesto se refleja como instrumento de planificación y control administrativo, en el que confluyen los objetivos de la empresa traducidos en refe- 
Factores clave de éxito en la gestión presupuestaria del sector pastas alimenticias Rodríguez Medina, Guillermo; Vílchez, Gil y Urdaneta Espinosa, Armando

rentes financieros y permite la asignación de recursos a los distintos centros de responsabilidad, mediante diversas técnicas y estrategias que responden a criterios integradores de una gestión empresarial presupuestaria.

En tal sentido, se pretende determinar los FCE en la gestión presupuestaria en el sector de pastas alimenticias en la región zuliana, para potenciar el éxito de los objetivos de la organización. En la práctica empresarial el sector bajo estudio, ha hecho énfasis en aspectos como el costo de fabricación, la calidad y sus costos en los procesos, el tiempo de manufactura y de entrega, y la productividad total y parcial de la planta, en tal virtud, serán abordados para determinar en cada uno de ellos los aspectos más relevantes, no obstante, antes de abordar dichos aspectos se considera pertinente tratar los fundamentos en que se basa la gestión presupuestaria.

El proceso presupuestario llevado a cabo en una organización atraviesa una serie de fases relacionadas con la administración. Burbano y Ortiz (2000), plantean al respecto cinco fases: Preiniciación, Elaboración del Presupuesto, Ejecución, Control, y Evaluación. Por otro lado, Welsch et al. (1994) establecen solo tres: Planeación, Dirección, y Control.

De lo expuesto por los autores anteriores, existen diferencias que marcan el enfoque llevado a cabo en el proceso presupuestario. Es así como Burbano y Ortiz (2000) distingue inicialmente las fases de Preiniciación y Elaboración, de manera separada dentro de la actividad presupuestaria, aspectos que Welsch et al. (1994) integra en una sola fase denominándola Planeación.
Otra de las fases es la ejecución, mencionada así por Burbano y Ortiz (2000), la cual difiere con relación a lo plasmado por Welsch et al. (1994), ya que éste último lo refiere como fase de Dirección, ampliando un poco más el alcance de ésta etapa, en virtud que no solo es la simple ejecución sino también la estructuración de planes por medio de la coordinación de diferentes programas operativos y financieros; y finalmente, para Burbano y Ortiz (2000) el proceso presupuestario concluye con el Control y la Evaluación, las cuales se desarrollan durante y al final de la gestión respectivamente, no así para Welsch et al. (1994), quienes afirman que el cierre del ejercicio presupuestario se consolida en una sola fase final: El Control, que permitirá la retroalimentación y replanificación del mismo.

Las fases presentadas por los autores anteriores, permite ser complementada, de acuerdo a los autores del presente trabajo, con la etapa de "Simulación”, y sistematizada de la siguiente manera: Preiniciación, Elaboración, Simulación, Ejecución, Dirección y Control. La fase de Simulación; consiste en el análisis de sensibilidad, con el objetivo de observar los efectos que pudieran provocar los cambios en las variables manejadas en el presupuesto dentro de diversos escenarios, para de esa forma sustentar racionalmente la toma de decisiones gerenciales y garantizar el éxito en la gestión presupuestaria.

La gestión presupuestaria está referida a las acciones que hay que llevar a cabo, aplicando la herramienta presupuestal como termómetro en el desarrollo de las diferentes actividades operacionales y financieras, a las que se le han asig- 
nado recursos para el cumplimiento de los objetivos trazados por la organización.

Entre las estrategias de la gestión presupuestaria está la determinación de los factores clave de éxito y hacerle seguimiento, a través de indicadores tanto financieros como no financieros, entre otros, para potenciar el éxito de la gestión.

\subsection{Formalidad vs. Informalidad del proceso presupuestario}

La formalización de cada una de las fases tratadas anteriormente es otro de los aspectos vinculados al buen desarrollo del proceso presupuestario, ello en virtud de facilitar una mayor objetividad en las disposiciones estratégicas emanadas de la gestión. Welsch et al. (1994) plantean que la formalización exige el establecimiento y la observancia de fechas límites para los actos de toma de decisiones, de planificación y control, así como, una base lógica para una flexibilidad racional.

De tal manera que, es importante que en la gestión presupuestaria se tomen en cuenta estos aspectos, los cuales por su margen de intangibilidad en muchos casos pasan por desapercibido. Ahora bien, ¿Qué es lo recomendable para las organizaciones en su gestión presupuestaria?; desde luego la interacción de los actores, que permitan el establecimiento de un sistema presupuestario efectivo e iniciar la formalización de los mecanismos para regular y fluir la comunicación e información de una manera más pertinente, oportuna y equilibrada, sin que ello represente un obstáculo o ca- misa de fuerza a las actividades normales de la organización, a fin de no extremar la formalización de los procesos.

El otro extremo de la formalización de las fases del proceso presupuestario, esta representado por el exceso de informalización, en virtud de la alta carga subjetiva provista por esta forma de operar, caracterizada por la falta de una orientación o desconocimiento claro de los objetivos que persigue la organización, y al abuso en la concentración de tareas y funciones administrativas menos relevantes. A estos efectos Welsch et al. (1994) comentan que la exageración en la formalización y una administración excesivamente informal implican serios peligros para la gestión presupuestaria, quizás aun de mayor trascendencia que la falta de formalización.

La formalidad del proceso presupuestario pareciera algo normal y corriente en las organizaciones, no obstante, la realidad de ciertas empresas del sector de pastas alimenticias en la región zuliana es otra, en la que muchas veces se impregna de informalidad a los procesos presupuestarios, reduciéndose la actividad presupuestal indebidamente a simples planes incrementales, basados en cifras de ejercicios anteriores sin ameritar un mayor análisis de las áreas clave dentro de la gestión presupuestaria. De acuerdo a Sweeny et al. (1984) refieren que el uso de esta técnica presupuestal es frecuentemente mediante tradicionales procedimientos de planeación y de presupuestación, centrándose los directivos principalmente en los cambios que se prevén en comparación con los niveles de gastos y/o ingresos del año anterior. 
Factores clave de éxito en la gestión presupuestaria del sector pastas alimenticias Rodríguez Medina, Guillermo; Vílchez, Gil y Urdaneta Espinosa, Armando

\subsection{Enfoques presupuestarios: incremental, base cero, y por actividades}

La gestión presupuestaria basada en el enfoque incremental, a menudo provoca problemas, tales como la imposibilidad de identificar o destacar problemas clave y áreas decisivas para la empresa, por lo que consecuentemente el empleo de este enfoque seria una desventaja para la organización que pudiera repercutir en la rentabilidad de la misma.

Otro enfoque presupuestal considerado en las organizaciones es el Presupuesto Base Cero (PBC), el cual es definido como "aquel que se realiza sin tomar en consideración las experiencias habidas" (Del Río González, 2002: I-22). Por su parte, Paniagua (1997:128) profundiza un poco más su conceptualización en torno al PBC y refiere que "es un proceso que estructura analíticamente (por capas, niveles o estratos) y que permite a la dirección de la empresa tomar decisiones sobre la eficiente asignación de recursos a los sectores de mayor rendimiento".

Las aseveraciones hechas por los autores anteriores son complementarias entre si, dado que permite afirmar que el PBC es un enfoque analítico formulado a partir de cero, y dirigiendo su atención hacia aquellas áreas que pudieran ser consideradas como clave en el éxito de la gestión presupuestaria, tomando en cuenta la relación costo beneficio.

Por otra parte, el enfoque de presupuesto basado en actividades (PBA), es un concepto relativamente moderno en la gestión presupuestaria que está siendo muy aplicado en el sector manufacturero, y se complementa con el PBC, pues, aquel hace énfasis en el proceso productivo y éste en el aspecto administrativo. En tal sentido, Kaplan y Cooper (1999: 295) manifiestan que: "Los PBA proporcionan a las organizaciones la oportunidad de autorizar y controlar los recursos que suministran basándose en las demandas previstas para las actividades realizadas". Mientras que Lorino (1996: 35) señala:

"es una nueva filosofía de gestión que se traduce en una panoplia completamente renovada de herramientas y de métodos, desde la medición de la eficiencia hasta la gestión preventiva de los recursos humanos, pasando por la gestión de los flujos de materiales, el análisis del valor y la evaluación de las inversiones".

De tal manera, el alcance de las prácticas en la gestión presupuestaria suele subestimar en muchas oportunidades las bondades de enfoques modernos presupuestarios. Si bien es cierto que la aplicación de enfoques tradicionales son empleados frecuentemente por las organizaciones, debido a la facilidad en su formulación, no es menos cierto que el uso de enfoques contemporáneos, como el $\mathrm{PBC}$ o el PBA hacen consideraciones más completas e integradas de los procesos presupuestales, que plantean la necesidad en las empresas de reformular su gestión presupuestaria dentro de un marco más formalizado y analítico, que le brinde los elementos de juicio necesarios para el fortalecimiento del éxito empresarial y permiten determinar con mayor facilidad los FCE en la gestión presupuestaria.

Ante esta realidad, es necesario que durante el desarrollo de la gestión 
presupuestaria se lleven a cabo acciones que permitan, hacer énfasis en las actividades, tanto administrativas como operacionales que son medulares en la organización, y proveerlas de recursos que faciliten el logro de los objetivos trazados y no desviarse a aquellas actividades que no son esenciales, es decir, no sacrificar tiempo y recursos en actividades superfluas.

\section{Factores clave de éxito en la gestión presupuestaria}

Hoy en día el sector de pastas alimenticias en la región zuliana no puede estar al margen de la competencia generada en la industria, en virtud de la confluencia de un sin número de nuevos actores en este mercado global, que conlleva a estas empresas a una permanente búsqueda de elementos que se constituyan en una ventaja competitiva y que coadyuven al asentamiento de las mismas en un nicho específico.

Esta realidad justifica per se que las organizaciones estén implementando estrategias de cambios fundamentadas para la identificación de los FCE en la gestión presupuestaria de las organizaciones del sector de pastas alimenticias, de acuerdo con los rasgos más recurrentes y pertinentes observados en el sector estudiado.

El estudio de los FCE en la gestión presupuestaria de las diversas empresas es un campo que ofrece múltiples alternativas, toda vez que depende del tipo de industria que se esté analizando y de las características de ese mercado en particular. La identificación clara y precisa de los FCE y su interrelación pueden llegar a ser sin lugar a dudas la diferencia de la competitividad y del éxito en la gestión presupuestal integral de cada negocio, y más aún dentro del marcado dinamismo que nos impone la globalización.

Los responsables de la gestión presupuestaria necesitan comprender el contexto de la industria para evitar un diagnóstico erróneo de las empresas que lo integran, en cuanto a los elementos fundamentales, para lograr el éxito de dicha gestión, no solo en el corto plazo, sino también garantizar el crecimiento sostenido de los resultados operativos y financieros en el largo plazo.

De acuerdo a Sweeny et al. (1984: 34), "la determinación de los factores importantes;consiste en comparar a los ganadores y a los perdedores de esa industria. Los atributos que tienen en común las empresas con éxito, y de los cuales carecen las que no lo tienen, son factores clave del éxito".

Estos aspectos señalados refieren nuevos paradigmas dirigidos a una gestión presupuestaria cada vez más integral y que se contrapone a esquemas tradicionales enfocados en una gerencia que hace énfasis sólo en procesos de control presupuestario simples y menos formales, procurando hacer énfasis en áreas medulares y calificadas como clave para el éxito de la dirección llevada a cabo en la empresa; dirección apoyada en una tecnología de información de vanguardia que le permita fundamentar la plataforma de las acciones presupuestarias desarrolladas con miras al cumplimiento de los objetivos financieros formulados.

Según Drucker (2000), la información puede llegar a transformar una gestión presupuestaria en referente a la polí- 
Factores clave de éxito en la gestión presupuestaria del sector pastas alimenticias Rodríguez Medina, Guillermo; Vílchez, Gil y Urdaneta Espinosa, Armando

tica empresarial; basándose en una tecnología de información flexible, que permita la integración de todas las fases del proceso presupuestario, y en la que el recurso humano tiende a ser reducido, selectivo, y con un mayor grado de especialización en virtud del incremento del elemento tecnológico.

Las innovaciones tecnológicas de la información ciertamente han repercutido en el manejo del proceso presupuestario, requiriendo para ello un recurso humano calificado, que sea capaz de interpretarlo y utilizarlo para el desarrollo de una buena gestión presupuestaria. De acuerdo a Rodríguez et al. (2005: 107),

"El recurso humano es parte crucial en la gestión presupuestaria, porque su capacidad, el compromiso, la persuasión, y la inteligencia que debe poseer la gerencia, son factores importantes para convertir el sistema presupuestario en medio que permita alcanzar el éxito de los objetivos trazados y no tomarlos como un fin en si mismo."

De acuerdo al estudio realizado los FCE más relevantes en el sector de pastas alimenticias fueron el costo de fabricación, la calidad y sus costos en los procesos, la productividad y el tiempo de manufactura y entrega, los cuales fueron estudiados para comprender los aspectos más importantes y su comportamiento.

\subsection{El costo de fabricación}

Convencionalmente la gerencia de forma regular ha utilizado indicadores de productividad, de rentabilidad sobre la inversión, estándares técnicos de rendimiento y de costo, el propio presupuesto, entre otros; éstos han sido instrumentos signados desde finales del siglo XIX, bajo el impulso de Frederick Taylor gestor de la denominada "administración científica", los cuales permiten medir el desarrollo de la gestión presupuestaria en cuatro principios, de acuerdo a Lorino (1996):

1. Los mecanismos de eficiencia son estables en el tiempo;

2. El directivo tiene una información perfecta sobre los mecanismos de eficiencia del sistema que dirige;

3. La eficiencia productiva se identifica con la minimización de los costos;

4. El costo global es equivalente al costo de un factor de producción dominante, generalmente la mano de obra directa.

Lo anterior dista enormemente de los cambios actuales en el parque industrial y particularmente del subsector de pastas alimenticias en la región zuliana. La gerencia frente a estos paradigmas ha visto las transformaciones de estos axiomas a lo largo del desarrollo de su gestión presupuestaria, en virtud que la aceleración de la innovación tecnológica, las evoluciones muy rápidas del mercado internacional y la creciente apertura de la competencia mundial, ya no permiten esperar que los mecanismos de eficiencia permanezcan estables a través del tiempo.

Por otro lado, está el hecho de que ya la mano de obra directa, que es uno de los elementos en la estructura de costo, ya no es un factor predominante debido al incremento voraz del uso de la tecnología tanto de hardware como de software, que al mismo tiempo le ha permitido a las empresas del sector de pastas alimenticias minimizar sus costos, y maximizar la eficiencia en su proceso productivo. En 
efecto, el estudio realizado al sector ubicado en el municipio San Francisco del estado Zulia, al preguntarse sobre la composición de la estructura del costo de fabricación en relación a sus elementos que lo integran (materia prima, mano de obra directa y carga fabril), como FCE, las tres empresas estudiadas señalaron que el elemento más importante, en cuanto a costo, es el de la materia prima, seguido por la carga fabril y por último la mano de obra directa.

El mercado de hoy en día se ha convertido en un mercado de demanda, donde los clientes cada día son más exigentes en cuanto a calidad, precios y la atención personalizada, que impulsan a las empresas del sector de pastas alimenticias de la región zuliana a adaptarse a nuevos requerimientos, principalmente en la disminución de los costos con un mayor valor, para de esa manera poder ser competitivos en el mercado.

Con respecto al costo de fabricación, Horngren et al. (1996: 6) comentan que; "Las organizaciones están bajo presión continua para reducir el costo de los productos o servicios que venden a sus clientes". Esto es el resultado lógico de una competencia cada vez más intensa en la búsqueda progresiva de ser más eficiente en el uso de los recursos y satisfacer las necesidades de consumo, especialmente en pastas alimenticias donde la estrategia de liderazgo en costos permite el posicionamiento del mercado y asegura su competitividad.

Por otra parte, Kaplan y Cooper (1999:267) señalan que "en los entornos altamente competitivos de hoy en día, no es suficiente con ser el más eficiente; también es necesario formar parte de la cadena de aprovisionamiento más eficiente".

La idea de ser competitivo debe reflejar un mayor alcance organizacional que se circunscriba aguas arriba y aguas abajo, tanto con los clientes internos como externos así como a los proveedores de la empresa a lo largo de esa cadena de aprovisionamiento, para crear un mayor valor económico agregado en relación con los activos regentados y del producto y/o servicio ofertado.

La gestión presupuestaria desarrollada por las empresas del ramo de pastas alimenticias está signada por la capacidad de utilizar estrategias acordes con el contexto en que se desenvuelven, específicamente las relacionadas con costo, para liderar y rendir de manera sostenida y permanente por encima de las expectativas del mercado. Al respecto Porter (2000: 30) señala que,

"el liderazgo en costo es tal vez la más clara de las tres estrategias genéricas

(...) los productores de costo bajo venden clásicamente un estándar, o un producto sin adornos y colocan un énfasis considerable en la escala de madurez 0 en las ventajas de costos absolutas de todas las fuentes."

La gestión presupuestaria del sector bajo estudio se halla basada en un liderazgo de costos, en virtud de las siguientes características:

- Precio de venta reducido: Al referirnos a productos de consumo masivo estándar, es notorio la necesidad del sacrificio de los márgenes de beneficio para una fabricación al mínimo costo. 
Factores clave de éxito en la gestión presupuestaria del sector pastas alimenticias Rodríguez Medina, Guillermo; Vílchez, Gil y Urdaneta Espinosa, Armando

- Producción a grandes volúmenes: Las plantas procesadoras de pastas alimenticias aprovechan el potencial de producción en razón de la existencia de la capacidad práctica, para penetrar en nuevos mercados.

- Atributos de rendimiento: Se venden más productos del ramo de pastas alimenticias destacando la consistencia, durabilidad, fácil preparación, y mayor economía.

Estas cualidades refieren que el resultado de la gestión presupuestaria se halla supeditado fundamentalmente a un perfil enfocado en la eficiencia de los recursos provistos basados en el costo, para su desempeño económico y a su vez clave para la competitividad y sostenimiento de las organizaciones empresariales de este sector industrial estudiado.

\subsection{La calidad y sus costos en los procesos}

La creciente integración de la economía mundial está obligando a la gerencia a prestar una mayor atención a la calidad de bienes y servicios ofertados en el mercado. La gestión presupuestaria de una compañía manufacturera se compone de un cúmulo de actividades (Compra, Producción, Almacenamiento, Ventas, entre otros), que conforman el compendio del sistema presupuestario que da soporte a dicha gestión. En tal sentido para que una gestión presupuestaria logre el mantenimiento de una ventaja competitiva sostenible en el tiempo es clave la consideración de la calidad como un FCE, ya que debe ser una norma competitiva que los gerentes han de aplicar de manera continuada.
La Comunidad Europea ha establecido normas formales de calidad, por ejemplo ISO 9000, 9001, 9002, entre otros, como línea de base para el diseño de productos y certificación de que éstos satisfacen las normas; las cuales indican si las plantas manufactureras y las organizaciones proveedoras de servicios ponen o no en práctica procedimientos esquematizados bajos estándares de calidad internacional.

En el caso particular, según las entrevistas aplicadas a las empresas de pastas alimenticias en la región zuliana, dos de ellas disponen de laboratorios de control de calidad especializados que han avanzado hacia programas de certificación para obtener verbigracia certificaciones ISO 9002, y en especial en la categoría correspondiente a los circuitos de producción, constituyéndose en un elemento adicional que garantiza la calidad de los productos del sector y minimiza los impactos financieros en el presupuesto, en virtud de la reducción de reprocesos, (costo de calidad), dada la implementación de las más estrictas normas de preparación y elaboración de las pastas.

De acuerdo a lo planteado por Horngren et al. (2002), los costos de la calidad se clasifican en Costos de previsión, examinación, de falla interna, y de falla externa. Por su parte, Hansen y Mowen (2003) mencionan una clasificación más general agrupándolas en actividades de control y actividades de fracaso, y explica que los costos de efectuar estas actividades se conocen como costos de calidad.

Al indagar en las empresas del sector, en relación a los costos de calidad en su gestión presupuestaria, el resultado 
obtenido señala que dos de las tres empresas analizadas consideraron más importante los costos de prevención en virtud de la importancia de los programas de capacitación de calidad, las auditorias de calidad, ingeniería de diseño, entre otros, que son clave para la competitividad de las mismas, la otra empresa bajo análisis manifestó los costos de fallas internas y los de fallas externas, a causa de los costos de reproceso y de las ventas no realizadas, por defectos en el producto o ajustes por reclamación, entre otros.

La práctica empresarial del sector de pastas alimenticias apunta hacia los siguientes aspectos: prevención, para evitar la elaboración de pastas cortas y largas que no estén ajustadas a las especificaciones estandarizadas; fallas internas, debidos a los reciclamiento de algunos insumos recuperables antes del proceso de secamiento y de llegada al stock de productos terminados; y fallas externas, incurridas una vez distribuidos al mercado mayorista, debido a la devolución de productos por vencerse su ciclo de vida o por estar en disconformidad con los estándares generales de los productos alimenticios, los cuales resultan posterior a la venta y son básicamente en transportación y soporte del cliente.

Ante esta realidad descrita los desafíos que hoy en día se están dando sobre la gestión presupuestaria de las empresas del ramo de pastas alimenticias se tornan cada vez más alrededor de los costos de calidad del producto, ya que "pueden ser sustanciales y una fuente de ahorro importante" (Hansen y Mowen, 2003:596).

Por su parte, Horngren et al. (2002) señalan que, muchas compañías de todo el mundo perciben igualmente la calidad como una importante ventaja competitiva, debido a que un enfoque de calidad reduce costos y aumenta la satisfacción del cliente, conociendo y evaluando lo que representa la calidad en el diseño y en el cumplimiento de sus productos y servicios ofertados. Por otra parte, según Ivancevich et al. (1997:19) manifiestan que:

“(...) la calidad puede medirse también en términos de dinero. Si sumamos los gastos por hacer las cosas mal, como las reparaciones, dar servicio al cliente, inspecciones, exceso de inventarios, horas extraordinarias no planeadas, cuentas por cobrar vencidas, avisos de cambio en ingeniería, notificaciones de cambios en las compras (...) El precio de la disconformidad (...) asciende a más o menos cinco veces el beneficio antes de impuesto (...)"

De lo anterior, se desprende concretamente que la gestión presupuestaria llevada a cabo en la actualidad no puede estar al margen del seguimiento de las normas generales de calidad tanto de carácter interno diseñadas por la propia empresa, como externo en el ámbito nacional e internacional, amen de los costos que ello involucra, toda vez, que las organizaciones independientemente del sector en que se encuentren se hallan inmersas en un mercado cada vez mas global, que les exige una gestión ceñida inexorablemente a un proceso integral de funciones bajo una cobertura total de calidad.

\subsection{La productividad: total vs. parcial}

El mercado venezolano cuenta con un importante consumo de pastas, después del mercado italiano, así lo afirma 
Factores clave de éxito en la gestión presupuestaria del sector pastas alimenticias Rodríguez Medina, Guillermo; Vílchez, Gil y Urdaneta Espinosa, Armando

Nobile, citado por: Rodríguez et al (2004: 47), quien señala que, "Venezuela ocupa el segundo lugar como consumidor de pastas alimenticias a nivel mundial, después de Italia". Por otro lado, se obtuvo que en el país europeo se consumen 24 kilos de pasta por persona al año, en nuestro país este índice de consumo per cápita alcanza los 12 kilogramos (Benítez, entrevista 2004).

El sector de pastas alimenticias de la región Zuliana se ha caracterizado por ser competitivo, debido a un proceso acelerado de incorporación tecnológica que ha impulsado grandiosamente la capacidad de producción de las empresas del sector estudiado. Rico (1992:185) explica que "la tecnología debe ser utilizada estratégicamente, no para brindar beneficios psicológicos abstractos; sino para mejorar la productividad y el servicio al cliente".

En efecto, ante los cambios notorios del mercado, el factor de la productividad ha sido otro FCE determinante dentro de la gestión presupuestaria del sector analizado, Horngren et al. (2002:485) manifiestan que, "la productividad mide la relación entre los insumos reales usados (en cantidades y en costos) y las producciones reales elaboradas. Cuanto menor sea la cantidad de insumos para una determinada cantidad de producciones, o cuanto más altas sean las producciones para una determinada cantidad de insumos, más alto será el nivel productividad", es por ello que, de acuerdo con los estudios realizados, la incorporación de nuevas líneas de producción en las empresas de pastas alimenticias para incrementar la productividad, se ha hecho evidente dado el aumento del promedio de producción de toneladas mensuales de las pastas cortas y largas, llegándose incluso a quintuplicar la producción presupuestada a lo largo de los últimos tres años (Villalobos, entrevista 2004).

Hansen y Mowen (2003: 636) refieren que, "La medición de la productividad es simplemente una evaluación cuantitativa de los cambios que se dan en ésta". Además, expresan que existen dos medidas de productividad: la parcial, la cual consiste en medir la productividad para un insumo, y la total, que evalúa la eficiencia de todos los factores inmersos en el proceso productivo, es decir, la productividad está relacionado con la obtención de los productos, en los términos de mediciones totales y parciales de mezcla y rendimiento de los insumos empleados para generarlo.

Al respecto, luego de consultar a las empresas del sector, en relación con la Productividad, el resultado arrojó que el $100 \%$ de ellas aplican medidas de productividad parcial y ninguna señaló la productividad total, con lo cual fue evidente, la impracticabilidad de enfoques de medición multifactoriales en las unidades económicas analizadas, quizás debido a la complejización en la lectura e interpretación de tales medidas de productividad.

Los estudios abordados indican que las tres empresas del sector determinan la productividad parcial, centrándose básicamente en las de tipo operacional, puesto que miden constantemente en las plantas procesadoras la relación de cantidades físicas usadas en relación a la producción obtenida, tal como la materia prima utilizada para la mezcla y rendimiento optima, de acuerdo con el grado de coc- 
ción esperado, las horas hombre directas empleadas por lotes de producción expresadas en promedios por procesos continuos de producción, y el grado de uso de la capacidad práctica de la planta, son ejemplos, de las medidas de productividad parcial usadas en el sector y que apuntalaron a una evaluación segmentada de tales factores.

Las nuevas exigencias del mercado de pastas alimenticias obligó a las empresas del sector a incluir dentro de sus planes presupuestarios medidas de productividad parcial, para determinar el requerimiento de ciertos recursos, especialmente de personal, como en mantenimiento mecánico y electromecánico, entre otros, según los flujogramas de procesos continuos, el establecimiento de estándares de productividad en los procesos, y un control sobre la materia prima procesada para la producción de pastas cortas y largas, en función del cumplimiento de los estándares de productividad de rendimiento $y$ mezclas esperados acordes con la producción ponderada presupuestada.

De tal manera que es clara la incorporación de la productividad como un FCE en la industria de pastas alimenticias, soportada con mejoras tecnológicas y la formación de un recurso humano calificado, que apuntala al crecimiento del sector, y la adecuación flexible de la gestión presupuestaria a una realidad global cada vez más competitiva.

\subsection{El Tiempo de manufactura y entrega}

El desarrollo de nuevos procesos de producción, al igual que la permanente adecuación de la capacidad práctica en las empresas del ramo de pastas alimenticias, está imponiendo cada vez menos tiempos en las actividades de procesamiento y estableciendo nuevos estándares de eficiencia en los recursos utilizados en la manufactura tanto de pastas cortas como largas, así como en dar respuestas a los clientes. A este respecto Heizer y Render (2001: 247) establecen que "El objetivo de estrategias de proceso es encontrar un camino para producir bienes y servicios que satisfaga los requerimientos del cliente y las especificaciones del producto, teniendo en cuenta el coste y otras limitaciones de la gestión"; por otro lado Horngren et al. (2002), señalan que las empresas en la actualidad perciben el tiempo como un factor clave de éxito, ya que al realizar las cosas más rápido facilita el incremento de los ingresos y la disminución de los costos, y plantean que las organizaciones necesitan medir el tiempo para poder manejarlo apropiadamente; mediciones operativas tal como basadas en el tiempo de respuesta al cliente o en el desempeño del tiempo, que elevan significativamente la competitividad en el mercado.

Los tiempos relacionados con las operaciones se han reducido hoy en día en más de 50 por ciento en cuanto a las actividades de secado y empacado del producto final, aplicando para ello tecnología de punta, verbigracia, secado a altas temperaturas, en el cual la pasta se seca a más de 100 grados centígrados, reduciendo de esta manera el costo de fabricación y afinando en última instancia las características de calidad organoléptica de la pasta para favorecer al consumidor (Villalobos y Benítez, entrevistas 2004). 
Factores clave de éxito en la gestión presupuestaria del sector pastas alimenticias Rodríguez Medina, Guillermo; Vílchez, Gil y Urdaneta Espinosa, Armando

Por otra parte, al indagarse en torno a los aspectos más importantes en relación con el tiempo como arma competitiva, se encontró que dos de las tres empresas se inclinaron en el tiempo de operaciones (manufactura), en virtud que la eficiente distribución de su proceso se debe preponderantemente a la reducción en el tiempo de fabricación, mientras que la otra restante indicó el tiempo de respuesta al cliente, dado que es de importante valor estratégico.

De estos resultados se desprende, por un lado la gestión presupuestaria del sector ha tenido que considerar la adopción de nuevos estándares de tiempo en el proceso productivo, cuyo resultado final ha derivado en el mejoramiento del tiempo de proceso de manufactura, particularmente en el recorrido interno de la materia prima en las líneas de producción, la preparación de las maquinas y en los ciclos del proceso de las pastas; y por otro, el tiempo de respuestas al cliente en relación con las fechas estimadas para entrega del producto fue de menor importancia; sin embargo, de acuerdo con Horngren et al. (2002), el tiempo de respuesta al cliente es un elemento clave para la competitividad en muchas industrias, con lo cual tal situación pudiera desfavorecer el posicionamiento en el mercado para algunas empresas del sector estudiado.

Del mismo modo, se destaca un impacto altamente positivo a nivel operativo, debido a que las inversiones que se presupuestaron, fueron realizadas por las empresas del sector en nuevas maquinarias y equipos, en función de la optimización de los parámetros de tiempo para elaborar productos con una mayor resistencia en la cocción y menos porcen- taje de disolución de materia prima, es decir se fabrican productos en un menor tiempo, lo que permite realizar una efectiva entrega al cliente y distribución al mercado, sin menoscabo de la calidad organoléptica de los productos alimenticios, toda vez que se elaboran con un incremento en su firmeza y durabilidad, y se generan menos desperdicios a nivel de la materia prima en su estado inicial de producción en las plantas procesadoras (Villalobos, entrevista 2004).

La formulación de programas de producción con la adopción de estas estrategias empresariales modernizadoras, en donde el tiempo se instituyó como eje central en el mejoramiento de los procesos, repercutió significativamente en la gestión presupuestaria del sector en estudio, a tal punto de ser considerado como un FCE determinante en la potencialización de la capacidad productiva, así como para el cumplimiento de los objetivos presupuestarios trazados, además de aportar beneficios tanto a las empresas del sector como a los consumidores, en cuanto al acabado, consistencia y calidad del producto en sus diversas presentaciones de pastas cortas y largas tales como spaghetti, vermicelli, regatones, caracoles, plumitas, macarrones, tornillos, entre otros, representando en definitiva un punto critico en la competitividad de estas organizaciones de consumo masivo.

\section{Conclusiones}

Los factores clave de éxito determinados en la gestión presupuestaria en esta investigación reflejan rasgos similares en las empresas del sector de pastas alimenticias de la región zuliana, que 
apuntan su atención en los costos de fabricación, la calidad y sus costos en los procesos, la productividad y el tiempo de manufactura y entrega; aspectos que a su vez, refieren consecuentemente a una serie de elementos estratégicos a considerar por la administración de las empresas del sector, con miras al fortalecimiento de ventajas competitivas y de liderazgos en el mercado.

Con relación al Costo de fabricación, el estudio realizado determinó que la mano de obra directa, que es uno de los elementos en la estructura de costo, ya no es un factor predominante debido al incremento voraz del uso tecnológico en el que se hallan inmersas las empresas del sector de pastas alimenticias, lo cual ha coadyuvado a minimizar sus costos, y a maximizar la eficiencia de los recursos incorporados a la producción, constituyéndose en aspecto estratégico en el establecimiento de planes y políticas financieras en las asignaciones presupuestarias para las empresas consideradas.

En cuanto a la Calidad y sus costos en los Procesos, se determinó que la práctica empresarial del sector de pastas alimenticias, además del cumplimiento de las normas de calidad, consideró tres aspectos relativos a los costos de calidad: los de prevención, para evitar fabricar pastas alimenticias no acordes a los estándares de calidad establecidos; los de fallas internas, en virtud del reutilizamiento de insumos recuperables antes del proceso de secado y de llegada al almacén de los productos acabados; y los de fallas externas, en virtud de la devolución de productos vencidos en su ciclo de vida o debido a que los clientes estaban en disconformidad con los estándares de los productos alimenticios. De todos ellos, los costos de prevención fueron los de más alto impacto en los planes presupuestarios, en virtud de la continua asignación de recursos para programas de capacitación de calidad, auditoria de calidad, ingeniería y pruebas de diseño, entre otros.

De la Productividad, se observó que el uso de las medidas en este aspecto empleadas, fueron de tipo parcial y a su vez centradas en aquellas de carácter operacional, es decir, orientadas a destacar aspectos no reflejados tradicionalmente en los estados financieros de las empresas, evaluando regularmente la relación de cantidades físicas tal como, la materia prima utilizada, según los parámetros de mezcla y rendimiento esperados, las horas hombre directas empleadas por lotes de producción, expresadas en promedios por procesos continuos de producción, y el grado de uso de la capacidad práctica de la planta.

Finalmente, en cuanto al Tiempo de manufactura y entrega, se conoció que el sector sufrió modificaciones importantes en los estándares de tiempo en el proceso productivo, cuyo resultado final derivó en la consideración del tiempo de fabricación como elemento medular del proceso operativo, y particularmente en lo relacionado al recorrido interno de la materia prima, en las líneas de producción, la preparación de las maquinas y en los ciclos de procesamiento de las pastas, todo ello apoyado en el uso de tecnología de punta que mejoró sustancialmente el secado y empacado final de las pastas alimenticias, facilitando también el cumplimiento de la entrega a tiempo a clientes y la reducción de los costos operativos totales. 
Factores clave de éxito en la gestión presupuestaria del sector pastas alimenticias Rodríguez Medina, Guillermo; Vílchez, Gil y Urdaneta Espinosa, Armando

\section{Referencias Bibliográficas}

Amat, Joan (1994). Control Presupuestario. España. Editorial Gestión 2000 Pp.173.

Burbano, Jorge y Ortiz, Alberto (2000). Presupuestos. Enfoque moderno de planeación y control de recursos. Colombia. 2da edición. Editorial McGrawHill. Pp. 376.

Del Río González, Cristóbal (2002). El Presupuesto. Generalidades-TradicionalÁreas y niveles de responsabilidadProgramas y Actividades-Base Cero, así como teoría y practica. México. 9na Edición. Editorial Ecafsa. Pp. VII-5.

Drucker, Peter (2000). Gestión del Conocimiento. SERIE HARVARD BUSINESS REVIEW. España. Ediciones Deusto. Pp 245.

Galindo, Mûnch y García, Lourdes (1987). Fundamentos de Administración. México. 3era Edición. Editorial Trillas. Pp 240.

Hansen, Don y Mowen, Maryanne (2003). Administración de Costos. Contabilidad y Control. México. 3ra Edición. Editorial Thomson Learning. Pp. 970.

Heizer, Jay y Render, Barry (2001). Direccción de la producción. Decisiones estrategicas. España. 6ta. Edición. Prentice Hall. Pp. 451.

Horngren, Charles; Foster, George y Datar, Srikant (1996). Contabilidad de Costos. Un enfoque gerencial. México. 8va Edición. Editorial Prentice Hall. Pp 970.

Horngren, Charles; Foster, George y Datar, Srikant (2002). Contabilidad de Costos. Un enfoque gerencial. México. 10ma Edición. Editorial Prentice Hall. Pp 906.

Ivancevich, John; Lorenzi, Peter; Skinner, Steven y Crosby, Philip (1997). Gestión
Calidad y Competitividad. España. Editorial McGraw-Hill. Pp. 785.

Kaplan, Robert y Cooper, Robin (1999). Coste y Efecto. Barcelona. 2da. Edición. Editorial Gestión 2000. Pp 401.

Lorino, Philippe (1996). El control de gestión estratégico. La gestión por actividades. México D.F. Editorial Alfaomega marcombo. Pp. 196.

Mallo, Carlos y Merlo, José (1995). Control de Gestión y Control Presupuestario. España. Editorial McGraw-Hill. Pp. 414.

Méndez, Carlos (1999). Metodología de la Investigación. Bogota. Colombia. Editorial McGraw Hill. Pp. 170.

Rico, Rubén (1992). Calidad Estratégica Total. Diseño Implementación y Gestión del Cambio Estratégico Imprescindible. Buenos Aires- Argentina. 2da. Edición. Editorial Macchi. Pp. 190

Rodríguez, Guillermo; Rodríguez, Belkis y Chirinos, Alira (2005). Sistemas de información en la gestión presupuestaria. Sector Pastas Alimenticias. Revista de Ciencias Sociales. Vol. XI. No. 1. Enero-Abril 2005. Universidad del Zulia. Venezuela. Pp. 105117.

Rodríguez, Guillermo; Chávez, Jorge; Muñoz Jesús y Rodríguez, Belkis (2004). Factores críticos en la gestión del proceso productivo en el sector de pastas alimenticias del municipio San Francisco-Estado Zulia. Revista Arbitrada Multidisciplinaria Multiciencias. Volumen 4. No. 1. Enero-Junio 2004. Universidad del Zulia. Venezuela. Paginas 46-55.

Ormaechea, Juan; Prieto, Marta y Escamilla, Juan (1998). Contabilidad de Gestión. España. Editorial Cultural. Pp. 320

Paniagua, Víctor (1997). Sistema de control presupuestario. México. 5ta Edición. 
Editorial Instituto Mexicano de Contadores Públicos, A.C. Pp. 148.

Porter, Michael (2000). Ventaja competitiva. Creación y sostenimiento de un desempeño superior. México. 10ma. Edición. Editorial Continental. Pp. 550.

Sweeny, Allen y Rachlin, Robert (1984). Manual de Presupuestos. México. Editorial McGraw-Hill. Pp.884.

Welsch, Glenn; Hilton, Ronald; Gordon, Paul (1994). Presupuestos Planificación y Control de Utilidades. México. 5ta Edición. Editorial Prentice Hall. Pp 697.

\section{ENTREVISTAS}

Benítez, Jairo. Jefe de Contabilidad en una de las empresas estudiadas. Maracaibo. 12-11-2004

Villalobos, Jorge. Gerente de planta en una de las empresas estudiadas. Maracaibo. 12-11-2004. 